\title{
Microstructure Formation and Toughening Effect of TiAl Based Microlaminate Sheet Deposited by Electron Beam HE Lu-ju ${ }^{1, a}$, MA Li $i^{2, b, *}$, SHAO Xian-yi ${ }^{3, c}$
}

\author{
${ }^{1}$ Education and Information Technology Center, Guangdong University of Petrochemical \\ Technology, Maoming 525000, Guangdong, China \\ ${ }^{2}$ College of Mechanical and Electrical Engineering, Guangdong University of Petrochemical \\ Technology, Maoming 525000, Guangdong, China \\ ${ }^{3} 1$ College of Physics \& Electronic Engineering, Taizhou University, Taizhou 318000, China \\ aheluju@gdupt.edu.cn, ${ }^{b}$ mali@gdupt.edu.cn, csxy8718@163.com
}

Keywords: EBPVD; Microstructure; Toughening; TiAl intermetallic; Mechanical properties

\begin{abstract}
A free-standing TiAl based microlaminate sheet with thickness of $300 \mu \mathrm{m}$ was deposited with high-power electron beam physical vapour deposition system. Microstructure and phase analysis of as-deposited samples were studied by XRD, SEM and TEM. Toughening by structure was analyzed with tensile test. The results show as-deposited sample was constituted of $\alpha 2-\mathrm{Ti} 3 \mathrm{Al}$ and $\gamma$-TiAl and $\alpha$-Ti. Alternation of rich aluminum zone and rich titanium zone are presented along cross section of TiAl depositing layer resulted from deviation of saturated vapor pressure between aluminum and titanium. After densification treatment, room-temperature tensile strength and elongation increased compared to that of as-deposited samples, reached $657 \mathrm{MPa}$ and 2.95\%, respectively. Tensile strength under high temperature exhibits an abnormal increase, exceeding 450 $\mathrm{MPa}$ at $1023 \mathrm{~K}$. The presence of ductile Ti layers can lead to cracks stagger along inter-laminar interfaces or crack deflection and the micro-bridge connection caused by ductile Ti layers, due to the $\mathrm{TiAl}-\mathrm{Ti} 3 \mathrm{Al} / \alpha-\mathrm{Ti}$ micro-laminate displaying a fine characteristic of delayed fracture.
\end{abstract}

\section{Introduction}

TiAl intermetallic, including $\alpha_{2}-\mathrm{Ti}_{3} \mathrm{Al}$ and $\gamma$-TiAl, has been studied extensively as substitutes for super-alloys owing to its lower densities and higher melting temperatures, which provide higher creep strength for structure materials including engines, turbine blades, panel of thermal proactive system $^{[1-2]}$. However, low temperature brittleness severely restricts their applications. One method to improve fracture resistance is reinforcing TiAl intermetallic by inserting ductile refractory metals to form multi-layer composite ${ }^{[3-4]}$. It was proposed originally by Kristic as a mean of increasing the energy dissipation in brittle materials ${ }^{[5]}$. Based on this idea, several multi-layer materials comprised of alternating layers of intermetallic and ductile metal (alloy) as Ti-6Al-4V/TiAl, NiAl/Ni, Nb/Nb $3 \mathrm{Al}$, have been prepared with different technologies including hot pressing, hot packed rolling, solid state diffusion welding ${ }^{[6-7]}$. Nevertheless, there are still some limitations by using these methods to rapidly prepare free-standing thin sheet with large size $(\varnothing>1000 \mathrm{~mm}, \delta<0.3 \mathrm{~mm})$. Electron beam physical vapor deposition (EBPVD) is a high-efficiency and non-equilibrium deposition technique. EBPVD is usually used to deposit thermal barrier coatings on blades and high-pressure turbine section vanes, which require surfaces with a smooth finish and ability to bear high thermo-mechanical strain. Recently, an amount of researches have been carried on EBPVD technology in the production of thin alloy sheets. $\mathrm{He}^{[8]}$ studied oxide dispersion strengthened super alloy with nanocrystalline surface layer prepared by EBPVD. $\mathrm{Li}^{[9]}$ analyzed isothermal oxidation behaviors of as-deposited and heat-treated Ni-11.5Cr-4.5Co-0.5Al sheet by EBPVD at 1074K. The objective of our work was to study the microstructure formation and how the structure of TiAl based microlaminate improve its room temperature toughness. 


\section{Experiment details}

L5 type EBPVD equipment made by Paton Welding Institute of Ukraine was applied in preparing TiAl based microlaminate thin sheet. Ti-48.5at\%Al and pure Ti ingot was used as vapor sources. Separated metallic targets were fixed in the water-cooled crucibles. During the depositing process, vapor was condensed on a rolling substrate above crucibles. Deposition was conducted in vacuum obtained by using a turbo-molecular pump. Following deposition the thin sheet were removed from their substrates and then hot-pressed under $1300 \mathrm{~K}$ under $150 \mathrm{MPa}$ pressure for two hours in protective atmosphere.

The structure of the alloys was examined by $\mathrm{X}$-ray diffraction with $\mathrm{Cu}-\mathrm{K} \alpha$ radiation and transmission electron microscopy (TEM). Microstructure observation was performed by a ZEISS optical microscope and S-4800 scanning electron microscope. Tensile tests were carried at 298-1023K. Tensile specimens were machined into I-shape with $20 \mathrm{~mm}$ in gauge length and were tested at room temperature and $1 \mathrm{~mm} / \mathrm{min}$ at high temperature.

\section{Results and discussion}

Microstructure and phase. Fig.1 (a) shows the microstructure of cross section of TiAl based microlaminate consists of 14 layers TiAl compound and 15 layers Ti, with a thickness of $300 \mu \mathrm{m}$. The mean component layer thickness ratio is about 1:1. To detect possible deformation induced diffusion linear EDX was done on sample which indicated that the contents of Aluminum and Titanium present periodic fluctuation along the deposition direction on cross section. Component scanning results show that the percentage composition of TiAl layer, interfacial region between component layers and Ti layers are Ti-49at\%Al, Ti-25at\%Al and Ti-1.2at\%Al respectively (Fig.1(b)), constituent of three distinct regions correspond to different phase compositions of $\gamma-\mathrm{TiAl}+\alpha_{2}-\mathrm{Ti}_{3} \mathrm{Al}, \alpha_{2}-\mathrm{Ti}_{3} \mathrm{Al}$ and $\alpha-\mathrm{Ti}$ based on Ti-Al binary phase diagram. Magnified cross view of the sample is displayed in Fig. 1(c), showing clear modulation structure and columnar grains in the component layer. It seems there are regular sub-layers in TiAl layer and formed alternating rich titanium region and rich aluminum region. This may be led by different saturated vapor pressure of aluminum and titanium: titanium atoms will fall behind aluminum atoms to evaporate and deposit onto the substrate surface. Zinsmeister has proposed an empirical parameter $\mathrm{K}$ which is used to characterize the ability of TiAl alloy to fractionate as described with formula (1):

$$
K=\frac{P_{\mathrm{Al}} \cdot f_{\mathrm{Al}}}{P_{\mathrm{Ti}} \cdot f_{\mathrm{Ti}}}\left(\frac{M_{\mathrm{Ti}}}{M_{\mathrm{Al}}}\right)^{1 / 2}
$$

Where $P_{\mathrm{Ti}}$ and $P_{\mathrm{Al}}$ are vapor pressures at the liquid alloy evaporation temperature; $f_{\mathrm{Ti}}$ and $f_{\mathrm{Al}}$ are activity coefficients of components titanium and aluminum; $M_{\mathrm{Ti}}$ and $M_{\mathrm{Al}}$ are molecular masses. In Eq. (1), if the parameter of $K$ is below 1 (when $K$ equal to unity, the alloy will be evaporated conformably), aluminum with higher vapor pressure will be evaporated firstly, and when the quantity of evaporated atoms increase enough, titanium with lower saturated vapor pressure will begin to evaporate. The greater of this difference is, the higher of the K parameter obtained from Eq. (1), and the higher is the initial aluminum concentration. Composition Changes in vapor phase lead to a concentration periodic variation along the condensates. Therefore, as long as evaporation time is long enough, the process will transit from original non-equilibrium transient state with changing composition into a transient equilibrium steady state with constant composition in the molten pool, steams and substrate. Zeng ${ }^{[11]}$ studied TiAl alloy evaporation and deposition by a mathematical model, which illustrated that it takes 960 seconds to achieve a steady stage when Ti-48at\%Al target surface temperature was $2000 \mathrm{~K}$. The deposition duration for TiAl layers was about 300 seconds in this work, and so deposition of TiAl layers was always in transient state which leads to continues fluctuation of composition in the molten pool, steam flow and condensate. Aluminum will be evaporated firstly during deposition process, and rich-aluminum particles in steam flow in the chamber will form rich-aluminum area on the condensate. Simultaneously, consumption of aluminum in the source will lead to rich-titanium on the surface of melting pool, thereupon the increasing 
taitanium content in the steam flow will transform from rich-aluminum to rich-titanium on the condensate. Ultimately, sub-layers formed by periodic variation of rich-aluminum region and rich-titanium region in TiAl component layer led by element content fluctuation of melting pool and steam flow. According to the thermodynamic calculation presented by Kattner ${ }^{[12]}, \mathrm{TiAl}_{3}$ phase formed in initial stage of deposition, and along with the deposition process, non-stable $\mathrm{TiAl}_{3}$ phase dissolved into TiAl phase and $\mathrm{Ti}_{3} \mathrm{Al}$ phase due to titanium diffusing into $\mathrm{TiAl}_{3}$ phase.
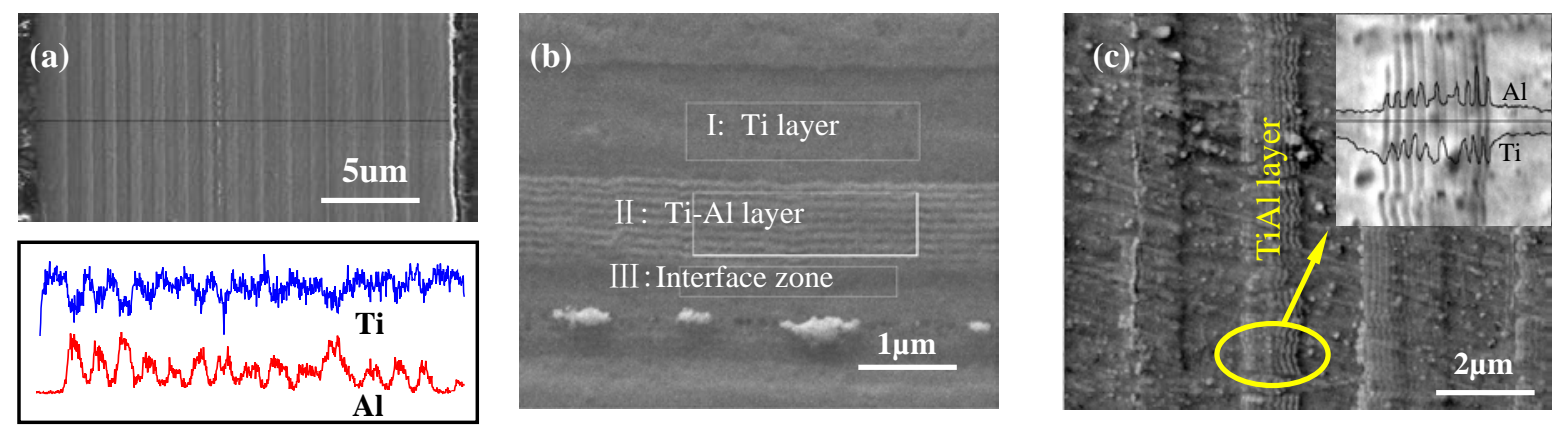

Fig.1. Microstructure and EDS analysis of TiAl based microlamiante sheet: (a) EDS line scan along cross section; (b) and (c) Amplified image of cross section

The XRD patterns also indicate that the composites comprised $\alpha-\mathrm{Ti}, \mathrm{Ti}_{3} \mathrm{Al}$ and $\mathrm{TiAl}$ phases, and crystal plane of diffraction peaks was given in fig.3. $\mathrm{Ti}_{3} \mathrm{Al}$ is a dominant phase in $\mathrm{TiAl}$ layer, whereas the characteristic peaks of TiAl present low intensity except higher peak intensity at $38.99^{\circ}$. By comparing EDS and analysis results with phase compositions ranges shown in the Ti-Al binary phase diagram, it was thought that due to the Al diffusion from intermetallic layer into adjacent Ti layer, there is a lower content of TiAl phase in intermetallic layer especially at interfacial region. Figure.4 shows a cross-sectional TEM micrographs of TiAl layer which confirms the presence of $\mathrm{Ti}_{3} \mathrm{Al}$ phase and $\mathrm{TiAl}$ phase. It can be found that $\mathrm{TiAl}$ phase with irregular streaky structure and $\mathrm{Ti}_{3} \mathrm{Al}$ particles were interspersed with non-flat rough interfaces. It is believed that the periodic variation of element content lead to the formation of $\mathrm{Ti}_{3} \mathrm{Al} / \mathrm{TiAl} / \mathrm{Ti}_{3} \mathrm{Al}$ two-phase architecture in $\mathrm{TiAl}$ component layers.

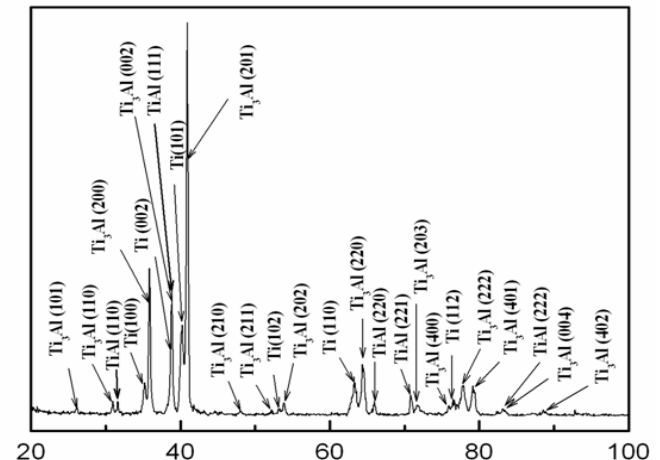

Fig.3.

Fig.2. X-ray diffraction pattern of TiAl based microlamiante sheet

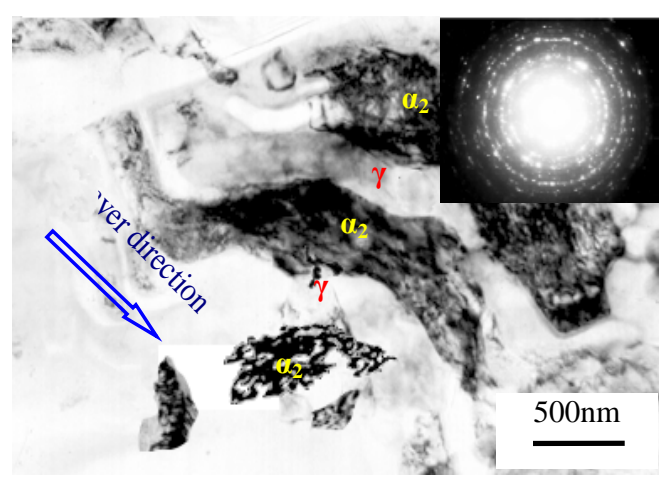

Fig.3. TEM micrographs on cross-section of TiAl based microlamiante sheet

Mechanical properties. Fig.4. shows tensile test results of TiAl based microlaminate at different temperatures. room-temperature tensile strength and elongation increased compared to that of as-deposited samples, reached $657 \mathrm{MPa}$ and $2.95 \%$, respectively. It can be seen that the stress-strain curve of single TiAl intermetallic deposited by the same technology exhibit linear elastic, while failure occurs almost without yielding. Correspondingly, the obvious stage of plastic deformation in stress-strain curve of TiAl based microlaminate indicates the improved property. Multi-stage and 
multi-stress peak value indicate that there initiates yield hardening during the tensile process. It is a main factor for deposited materials to have a low failure extension ratio that there exists high defect concentration and loose columnar crystals. However, the tensile curve of hot-pressed sample has a broad stage interval with a gentle variation. Hot-pressed technology can reduce large quantity of micro-pores, which create stress concentration effect and increase crack propagation resisting force by affording more effective loading area. So the breaking strain has been raised for a wide range. And then with temperature increasing, both elastic modulus and yield point increase abnormally. Furthermore, the breaking strength at $1023 \mathrm{~K}$ is even more than that at $823 \mathrm{~K}$. Elongation percentage data implicit brittleness at room temperature of TiAl intermetallic has been improved clearly due to the toughening by adding Ti layer. Tensile curves at high temperature have more obvious common specificity of saw-tooth form with many peak points. And slope coefficient of tie line between two adjacent peak points gradually reduces as strain rises. When pure Ti deforms, softened extent led by dynamic recovery recrystallization exceeds strain-hardening extent. The abnormal lifting of modulus and strength is definite relevant with Ti-Al intermetallic which has the abnormal temperature characteristic. Kavatata et al. investigated positive temperature dependence of the yield stress in TiAl intermetallic compound at $293 \sim 1273 \mathrm{~K}$ and concluded that pinning effect caused by dislocation slip at high temperature contribute to this abnormal yield behavior ${ }^{[13]}$.

TiAl based microlaminate sheets have special fracture behavior due to its particular microstructure. It is different for the crack propagation mode of micro-laminated foil with that of bulk materials in which the crack would extend along a straight line. For example, the crack propagation is not straight across the cross section, but staggering along interface or in layer for many times as shown in Fig.5. After roughly $40 \mu \mathrm{m}$ of crack growth, the macroscopic crack-path morphology shows a single dominant crack with limited branching and Ti layers behind the crack tip, thereby creating a bridging zone in the crack wake. According to material mechanics theory, stress subjected to superficial Ti layer is higher than that of inward Ti layer. So with increasing load, the outermost Ti layer will yield and failure first. During the loading process, crack will extend across laminar interface with high stress. In the meantime, brittle TiAl layer will break and form mouth-displacement to be cooperative deformation with Ti layer. As a result, inner cracks expand to the core by bridge connection mode. Failure of one or several Ti layers lead to redistribution of stress and stress increase in each layer, residue Ti layer can gradually restore load-supporting through strain-strengthening. When the above process carry on, tensile curve will form an irregular saw-shape till a descending trend lead to final failure as strain-strengthening can not make up for the strength loss by strain strengthening.

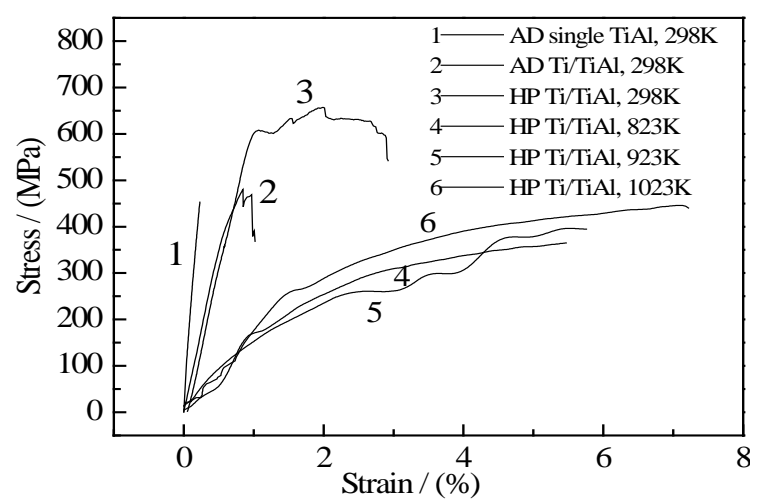

Fig.4. Tensile stress-strain curves of single TiAl alloy and TiAl based microlamiante sheet at different temperature

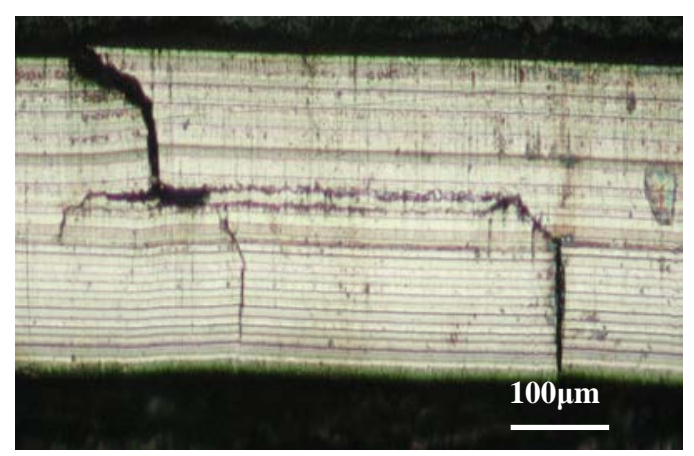

Fig.5. OM micrographs of tensile fracture surface of hot-pressed TiAl based microlamiante at $298 \mathrm{~K}$

Toughening effects. Fig.6. shows tensile fracture surface of TiAl based microlaminate specimen. It can be seen that as-deposited samples have clear laminate structure and inter-laminar interface. Smooth surface presents brittle rupture trait. Multi-cleavage plane with different height intersected and formed broad cleavage stage as shown in Fig.6 (a). In fact, those streams are formed by 
connecting with crack through some certain cleavage stages, and crack origin can be found by stream flow direction. It is shown that downstream direction is vertical to the laminar-interface, so the crack source of TiAl layer lies in the high stress zone of interface. Fig.6 (b) exhibits a dissimilar pattern compare to Fig. 6(a). There are both dimples and many cleavage planes on the fracture surface. Dimples come from aggregation of micro-pores. There will form micro-pores under stress in the materials, then those pores grew up and interlink under the effect of slipping. As temperature increases, size and number of dimples increase gradually, and the tensile samples revealed hybrid fracture. Fracture surface at $823 \mathrm{~K}$ presents obvious step-like and roughness pattern accompany with certain necking (Fig. 6(c)). Crack expansion proceeds with along interface and through interface alternatively. At $923 \mathrm{~K}$, quantity of dimples and pores increased at meantime and snake sliding and deflection along the grain boundary can be seen from the upper right of Fig.7(d). Since materials are composed of polycrystalline, crystal grains with different orientations will restrict each other. It is inevitable that slipping proceeds along multiple slip system at high temperature, and finally reflects on snake sliding trait by intersection of slipping system. When it reached $1023 \mathrm{~K}$, dimples spread all over the fracture surface (Fig. 6(e)). Abnormal strengthening of TiAl and softening of Ti resulted in lower coordinated deformability, and thus different layer would twist under load. Moreover, it can be seen from left side that several pores leaved over after some columnar crystals were whole pulled out which indicates binding force has been impaired under this temperature. Metallographic sections of crack path and fracture surfaces indicate that the higher toughness in the present TiAl based microlaminate sheet can be attributed to the effectiveness of Ti layers in promoting crack resistance and resultant crack bridging, cracks will continue for a certain distance along interface and then enter into the next layer as zone of a zone (Fig. 6(f)). The deflection of crack can absorb energy to improve fracture toughness property. Besides, it can be seen that there exist several concurrent zigzag cracks in the mark of a, b and c regions (Fig. 6(f)). Those cracks were blocked by Ti layer during the courses of expansion. Some of the Ti layers necked down and fracture surface exhibited a dimpled appearance, as well as the intergranular fractures, many transgranular cleavage cracks were also observed in the TiAl layers.
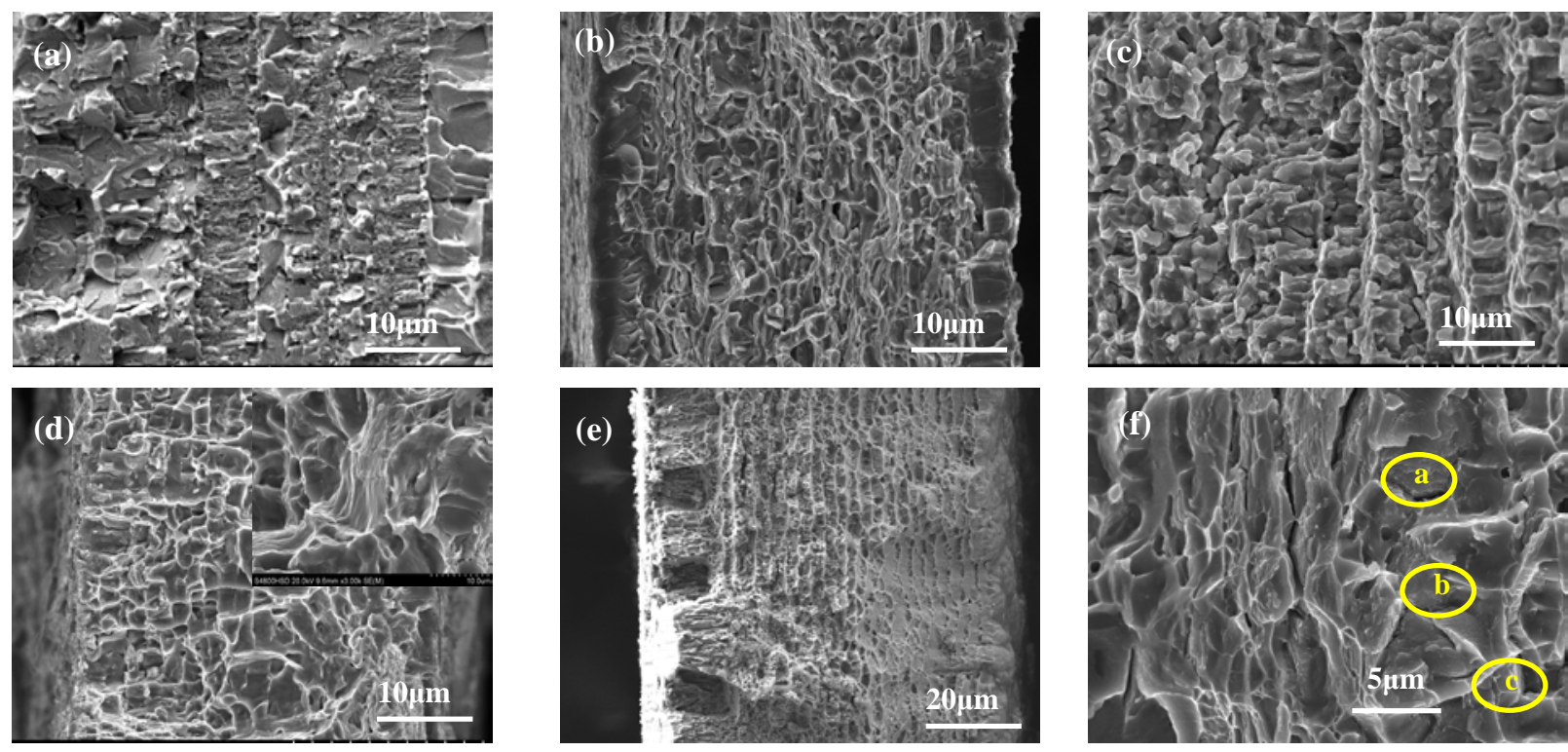

Fig.6. SEM micrographs of tensile fracture surface of TiAl based microlamiante at different temperature: (a) as-deposited, 298K; (b) hot pressed, 298K; (c) hot pressed, 823K; (d) hot pressed, 923K; (e) hot pressed, 1023K; (f) hot pressed, 298K. 


\section{Summary}

A free-standing TiAl based microlaminate sheet with thickness of $300 \mu \mathrm{m}$ comprising 15 layers Ti and 14 layers $\mathrm{TiAl} / \mathrm{Ti}_{3} \mathrm{Al}$ was prepared with EBPVD system. Component showed an alternating ups and downs along the normal direction of TiAl layers and results in several sub-layers due to the deviation of saturated vapor pressure between $\mathrm{Ti}$ and $\mathrm{Al}$ element. Addition of Ti layer into TiAl layer can increase the room temperature strength and percentage elongation distinctly compared to that of single TiAl intermetallic. The room temperature tensile strength value and percentage elongation of TiAl based microlamiante sheet after hot pressing densification process reached $657 \mathrm{MPa}$ and 2.95\%, respectively. Toughening effect TiAl based microlamiante sheet was attributed to the crack deflection and micro-bridge connection caused by the toughening $\mathrm{Ti}$ layers which can increase crack propagation resistance.

\section{Acknowledgements}

The support from the Maoming Science and Technology Project (Grant No. 915325), the Talent Recruitment Foundation from Guangdong University of Petrochemical Technology (Grant No. 650119), the Taizhou Science and Technology Project (Grant No. 14GY02) are highly appreciated.

\section{References}

[1] P. Raluca, F. Simone and S. Michael: Intermetallics Vol.56 (2015), p.1

[2] E. Schwaighofer, H. Clemens, S. Mayer, et al.: Intermetallics Vol.44 (2014), p.128

[3] M. Goral, L. Swadzba, G Moskal, et al.: Intermetallics Vol.19(2011), p.744

[4] R. B. Zhang, Y. Y. Zhang, Q. Liu, et al.: Materials Characterization Vol. 95(2014), p.157

[5] V. D. Krstic and M. D. Vlajic: Acta Metallurgica Vol.31(1983), p.139

[6] D. R. Bloyer, K. T. Venkateswara and R. O. Ritchie: Materials Science and Engineering A Vol.216(1996), p. 80

[7] H. Y. Kim, D. S. Chung, S. H. Hong: Materials Science and Engineering A Vol.396 (2005), p. 376

[8] X. D. He, Y. Xin, M .W. Li: J. Alloy Compd Vol. 461-470(2009), p.347

[9] M .W. Li, G. Zeng, F. He: J. Alloy Compd Vol.467 (2009), p. 30

[10]B. A. Movchan and G. S. Marinski: Surf. Coat. Tehcnol Vol.100-101 (1998), p.309

[11]G. Zeng, M .W. Li, Q.T. Zhang: Rare Metal Mat Eng Vol. 36 (2007), p.1759

[12]R. G. Reddya, A. M.Yahya, L.Brewer: J. Alloy Compd Vol.321 (2001), p.223

[13]T. Kavatata, T. Kanai, O. Izumi: Acta Metall Vol.33(1985), p.1355 